\title{
Courtship songs of Drosophila pseudoobscura and D. persimilis. II. Genetics of species differences
}

\author{
MARCUS A. WILLIAMS, AMANDA G. BLOUIN \& MOHAMED A. F. NOOR* \\ Department of Biological Sciences, Louisiana State University, Baton Rouge, LA 70803, U.S.A.
}

\begin{abstract}
Although male courtship songs have been repeatedly implicated in sexual isolation between numerous Drosophila species, no genetic studies have evaluated the genetic basis of differences between species beyond using quantitative genetic analyses of hybrids or surveying associations of song characters to five or fewer genetic markers. Here, we dissect the genetic basis of the difference between $D$. pseudoobscura and D. persimilis in two courtship song elements (interpulse interval and intrapulse frequency) using 15 molecular markers. We also evaluate the association between song elements and sexual isolation in these backcross hybrid males of these species. We find that song differences between these species are associated with at least two or three genomic regions, and the species difference in interpulse interval may be oligogenic. Courtship song differences are especially strongly associated with two inversions that differentiate these species. Further, we found that interpulse interval is strongly associated with mating success to $D$. pseudoobscura females, while intrapulse frequency is associated with mating success to D. persimilis females. Implications of these findings are discussed.
\end{abstract}

Keywords: courtship song, Drosophila persimilis, Drosophila pseudoobscura, sexual isolation, speciation.

\section{Introduction}

Males in many Drosophila species vibrate their wings in a species-specific manner prior to attempting to copulate with a prospective mate. Sound pulses emitted from this behaviour create what is referred to as the courtship song. Courtship songs are highly evolutionarily labile, sometimes diverging between taxa before the evolution of sexual isolation and hybrid sterility (Gleason \& Ritchie, 1998). Correspondingly, there are typically large differences between the courtship songs of closely related Drosophila species (e.g. Ewing \& Bennet-Clark, 1968; Chang \& Miller, 1978; Cowling \& Burnet, 1981; Geng et al., 1989; Hoikkala et al., 1994; Ritchie \& Gleason, 1995). Females of many Drosophila species appear to use certain courtship song traits in selecting among potential mates (Kyriacou \& Hall, 1982; Tomaru et al., 1995; Ritchie et al., 1998; Ritchie et al., 1999), and divergence in these traits between isolated taxa may ultimately lead to speciation.

Despite evidence of their significance, the genetic basis of differences between Drosophila species in courtship

*Correspondence. E-mail: mnoor@1su.edu song is not well understood. Previous studies of courtship song differences between species used either quantitative analyses of hybrids or utilized five or fewer marker loci per backcross (e.g. Ewing, 1969; Kawanishi \& Watanabe, 1981; Tomaru \& Oguma, 1994; Pugh \& Ritchie, 1996; Noor \& Aquadro, 1998; Hoikkala et al., 2000), resulting in generally low resolution. Recent advances in developing codominant molecular genetic markers (e.g. Hamilton et al., 1999) have made it possible to genetically dissect species differences in courtship song and other traits more thoroughly, and thus better understand the genetic changes associated with speciation.

Here, we examine the genetic basis of courtship song differences between Drosophila pseudoobscura and $D$. persimilis. These species have been used extensively in studies of speciation in general and sexual isolation in particular (Dobzhansky, 1936; Orr, 1987; Noor, 1995; Noor, 1997b; Wang et al., 1997). Males of these species court indiscriminately, but females exercise strong species discrimination (Merrell, 1954; Noor, 1996). Although discrimination between some Drosophila species is sometimes mediated by differences in cuticular hydrocarbon profiles (e.g. Coyne et al., 1994; Stennett \& 
Etges, 1997), D. pseudoobscura and D. persimilis females do not discriminate against heterospecific males using known hydrocarbon differences (Noor \& Coyne, 1996). Hence, we have begun to investigate their courtship songs as possible species recognition cues.

$D$. pseudoobscura and D. persimilis differ in at least two courtship song parameters: the interpulse interval (IPI) and the intrapulse frequency (Waldron, 1964; Ewing, 1969; Noor \& Aquadro, 1998). Both of these characters are associated with mate choice in other Drosophila species (Kyriacou \& Hall, 1982; Tomaru et al., 1995; Hoikkala et al., 1998; Ritchie et al., 1998; Ritchie et al., 1999). Previously, we documented variability among strains within these species in IPI and an effect of ambient temperature on both song parameters (particularly IPI, Noor \& Aquadro, 1998). We detected an association between intrapulse frequency and hybrid male mating success to $D$. persimilis females, but we failed to find an association between either courtship song parameter and mating success to D. pseudoobscura females in the small number of females tested. Our preliminary quantitative analysis suggested that both song parameter differences were controlled by both $\mathrm{X}$-chromosomal and autosomal loci, in contrast to the results of an earlier study in these species (Ewing, 1969).

We extend our previous study by examining the genetic basis of courtship song differences in backcross hybrid males using 15 codominant molecular markers (microsatellites and RFLPs). These markers span the five chromosomes of these species including the dot chromosome, which has never been examined for courtship song quantitative trait loci (QTLs) in any species. In addition, we evaluate the association between courtship song parameters and backcross hybrid male mating success to females of both species.

\section{Materials and methods}

\section{Stocks}

We maintained flies on a standard dextrose-yeast-agar medium in an incubator kept at $20^{\circ} \mathrm{C}$ on a $12: 12$ lightdark cycle. All flies were stored for eight days posteclosion. Males were separated the day before matings to minimize the effects of male crowding on mating success (Noor, 1997a).

We crossed females from an inbred stock of D. pseudoobscura derived from a 1993 collection in Flagstaff, Arizona (3rd chromosome inversion Arrowhead), to males from an inbred stock of $D$. persimilis derived from a 1993 collection in Mount St. Helena, California (3rd chromosome inversion Standard). These stocks have been used extensively in behavioural assays (Noor, 1995; Noor, 1996; Noor, 1997b; Noor \&
Aquadro, 1998). Hybrid female offspring were crossed to Flagstaff $D$. pseudoobscura males to produce the 'backcross males' assayed in these studies. Although $\mathrm{F}_{2}$ males may have been more useful for mapping, these males could not be produced because the $F_{1}$ hybrid males of these species are sterile. Only backcrosses to D. pseudoobscura were performed.

$D$. pseudoobscura and $D$. persimilis differ by chromosome inversions on the left arm of the X-chromosome, the right arm of the X-chromosome, and the centre of the second chromosome. In addition, both species are polymorphic for inversions on the third chromosome. We have selected strains with third chromosome arrangements that are likely to be the most common within these two species. Recombination is effectively inhibited within these inversions, but regions outside the inversions recombine readily (Noor \& Smith, 2000).

\section{Mating experiments}

We paired single backcross hybrid males with single pure species females in an Insectavox (Gorczyca \& Hall, 1987) recording device at room temperature $\left(21 \pm 1{ }^{\circ} \mathrm{C}\right)$. We focused our analyses on the high-rate repetition (HRR) song of these species if multiple song types were noted. Half of the males were paired with D. pseudoobscura females, and half were paired with D. persimilis females. Pairings were done between 9.00 AM and 11.30 AM immediately after being taken from the incubator. Approximately 15 pairings were recorded per morning, and copulations were noted if they occurred within five minutes. Each male was used in only a single pairing. We then froze males and extracted their DNA from single fly squash preparations (Gloor et al., 1993).

\section{Genotyping}

Microsatellite primer sequences, distances between markers, and variability of these markers within both species are presented elsewhere (Noor \& Smith, 2000; Noor et al., 2000a). Of these markers, we used DPSX004, DPSX009, DPS2005, DPS2001, DPS2002, DPS2006, gld, DPS2003, DPS3004, DPS3002, DPS3005, DPS4003, and DPS4002 (see Fig. 1 for autosomal map). DPSX004 (left arm of X-chromosome) and DPSX009 (right arm of X-chromosome) are freely recombining from one another, though recombination among loci within these chromosome arms is largely inhibited (Noor \& Smith, 2000). Other microsatellite loci localized within inversion differences between the species are noted on the figure. From these markers and the inversion differences that separate the strains used, we have virtually complete coverage of the $\mathrm{X}, 2,3$, and 5 chromosomes. There are 


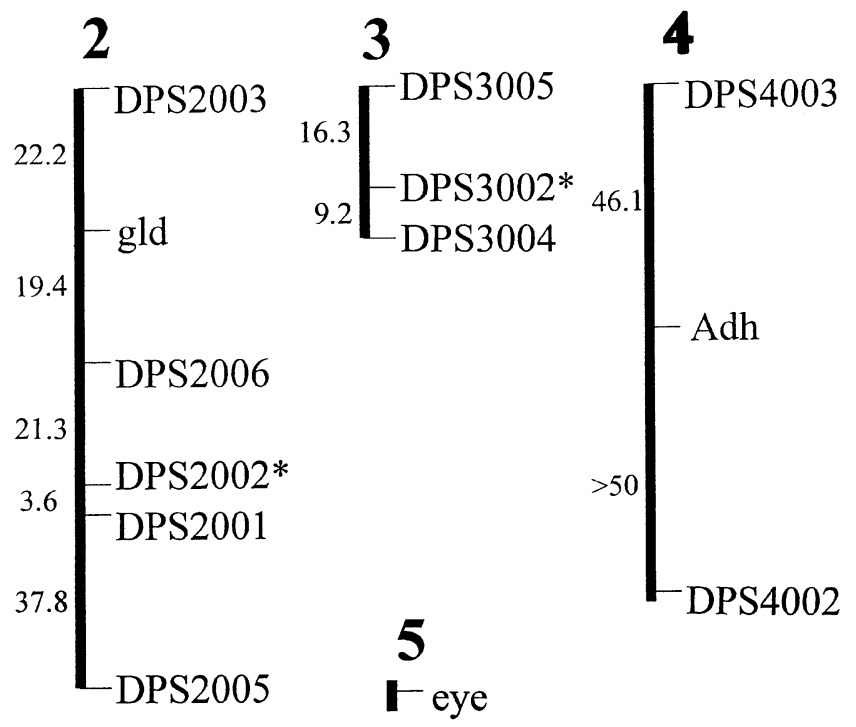

Fig. 1 Recombinational map of autosomal markers used in this study in hybrids of $D$. pseudoobscura and $D$. persimilis from data published in Noor \& Smith (2000). All autosomes are telocentric, and the centromere would be at the top of the figure for each chromosome. Markers within inversions that differentiate the strains used are designated with an asterisk. Recombinational distances were slightly different in this study, though the backcross was done in the same direction: see Figs 3 and 4.

unsurveyed areas on chromosome 4, however, due to scarcity of markers available on this chromosome.

We scored individual males as homozygous for the D. pseudoobscura allele or heterozygous for the D. pseudoobscura and D. persimilis alleles at each of the loci examined. We assayed microsatellite genotypes on agarose gels if the difference in size between the $D$. pseudoobscura and D. persimilis inbred line alleles exceeded 10 basepairs or on acrylamide gels for smaller size differences. For microsatellite assays on acrylamide gels, one primer bore an M13 complement tail at the $5^{\prime}$ end. We performed PCR in a $10-\mu \mathrm{L}$ reaction volume with 0.5 picomoles of each primer, 0.4 picomoles fluorescent-dye labelled M13 primer, $200 \mu \mathrm{M}$ dNTPs, $1 \mu \mathrm{L} 10 \times$ buffer $(100 \mathrm{~mm}$ Tris $\mathrm{pH} 8.3,500 \mathrm{~mm} \mathrm{KCl}$, $15 \mathrm{~mm} \mathrm{MgCl}_{2}$ ), $1 \mathrm{U}$ Taq polymerase, and $1 \mu \mathrm{L}$ from a $50-\mu \mathrm{L}$ single fly squash preparation. Cycling followed standard touchdown temperatures and times (Palumbi, 1996). Following PCR, $3 \mu \mathrm{L}$ of LiCor stopping buffer was added to the reactions, and $1 \mu \mathrm{L}$ was loaded onto an acrylamide gel (National Diagnostics Sequagel) on a LiCor 4200 DNA sequencer for documentation.

Microsatellite assays on agarose gels followed a similar PCR protocol except that primers were not fluorescently labelled and 5 picomoles of each primer were used per reaction. Following PCR, $3 \mu \mathrm{L}$ of Ficoll loading dye was added to the reactions, and all $13 \mu \mathrm{L}$ were loaded into a $2 \%$ agarose gel stained with ethidium bromide.

We also used two RFLP markers in our study. The Alcohol dehydrogenase (Adh) gene, located on the fourth chromosome, was amplified using primers 5'-TAGTTGTCAGGTGCAGCAGG-3' and 5'-ACGTTTTACAACTGGAACTGCG-3'. The eyeless gene, located on the dot chromosome, was amplified using primers 5'-ACTTCACAGGTTGTACAGTAATGTGTACC- $3^{\prime}$ and 5'-GTAGGTCGAGGCTATGAGGTCG-3'. We cut the $A d h$ reaction mixture with the restriction enzyme Pst $\mathrm{I}$, which cuts a 103-bp fragment off of the $D$. persimilis allele but had no effect on the 191-bp D. pseudoobscura allele. The eyeless reaction mixture was cut with the restriction enzyme Hinfl, which cuts a 100-bp fragment off of the D. pseudoobscura allele and a 97-bp fragment off of the $D$. persimilis allele. We assayed $A d h$ alleles on a $2 \%$ agarose gel stained with ethidium bromide, and eyeless alleles were assayed on our LiCor DNA analyser.

Backcross males exhibited the expected ratio of 50\% heterozygous : 50\% homozygous for all autosomal markers assayed. The D. pseudoobscura allele of DPSX004 was observed more often than the D. persimilis allele among these backcross males, though (approximately 2:1). This observation probably results from the frequent inability of backcross males bearing a D. persimilis X-chromosome to court (Noor, 1997b), hence preventing song recordings from these males.

\section{Statistical analysis}

We used CANARY sound analysis software (see Acknowledgements) to analyse the courtship songs of 508 backcross male flies, $14 \mathrm{D}$. pseudoobscura males, and $13 \mathrm{D}$. persimilis males. Interpulse interval and intrapulse frequency were measured directly from waveform diagrams of the song (see Fig. 2). We defined interpulse interval as the length of time from the beginning of one pulse to the beginning of the next pulse. Intrapulse frequency was defined as the inverse of the time between the first two peaks within a pulse. Males typically produced sound bursts with approximately 20 pulses. Median values of IPI and frequency were presented for each fly.

We used the QTL CARTOGRAPHER suite of programs (Basten et al., 1999) to analyse the genetic data. Individual male median IPI and frequency values were logtransformed to approach normality. We evaluated all markers for association with song traits by single marker regression (LRMAPQTL) and permuted traits 5000 times to evaluate statistical significance. We have conservatively applied a Bonferroni correction for 
Fig. 2 Courtship song of Drosophila persimilis showing pulses.

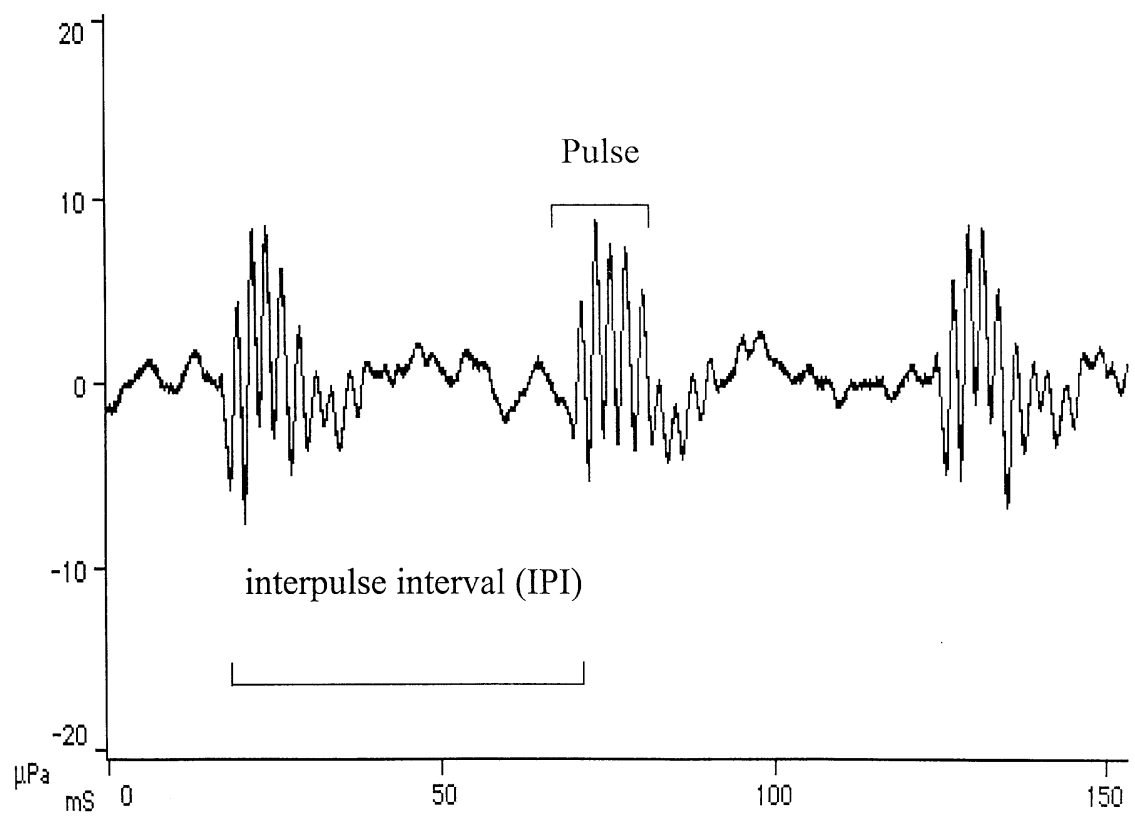

multiple comparisons (Rice, 1989) to our dataset, such that our threshold for significance is $\alpha=0.0033$ ( 0.05 divided by 15 markers). All statistically significant associations between markers and phenotypes were in the expected direction: males bearing a $D$. persimilis allele had higher courtship song IPI and frequency than males homozygous for $D$. pseudoobscura alleles. We conducted composite interval mapping analyses for linked markers to localize putative QTLs, using a conditioning window of $10 \mathrm{~cm}$. The threshold for significance in this interval analysis was estimated by permuting traits relative to genotypes 1000 times. Composite interval mapping analyses were conditioned on markers shown to have strong associations with the phenotypes in the single marker regression analyses.

We estimated the environmental variance $\left(V_{\mathrm{E}}\right)$ associated with courtship song parameters to be equivalent to the mean of the phenotypic variances of these song parameters in the pure-species inbred (isogenic) lines. This calculation assumes that no genetic variance in courtship song exists within these inbred lines, which seems to be a reasonable assumption. If we also assume $V_{\mathrm{E}}$ to be similar in pure species and backcross progeny, an overestimate of the total genetic variance $\left(V_{\mathrm{G}}\right)$ for courtship song parameters in backcross progeny would be the difference between the observed phenotypic variance $\left(V_{\mathrm{P}}\right)$ of song parameters in the backcross progeny and $V_{\mathrm{E}}$ in pure species lines. This figure overestimates $V_{\mathrm{G}}$ because genotype-environment interactions are lumped with genetic variance. The broad sense heritability $\left(H^{2}\right)$ for particular song traits in this backcross can then be estimated as $V_{\mathrm{G}} / V_{\mathrm{P}}$. In addition, the proportion of $V_{\mathrm{P}}\left(\% V_{\mathrm{P}}\right)$ associated with particular QTLs is provided in the composite interval mapping output from QTL CARTOGRAPHER (Basten et al., 1999). Finally, we derive a minimum estimate of the proportion of genetic variance $\left(\% V_{\mathrm{G}}\right)$ associated with a particular marker as the percentage $V_{\mathrm{P}}$ associated with the QTL divided by $H^{2}$. This figure may be a conservative minimum estimate of percentage $V_{\mathrm{G}}$ because $V_{\mathrm{G}}$ was overestimated (see above), and because dominance and epistatic genetic variance may not be identified.

We performed Mann-Whitney $U$-tests to determine if song characteristics were associated with mating success of backcross males to females of each species. For example, if song characteristics contribute to mating discrimination in these species, we would expect backcross males that mated with $D$. pseudoobscura females to have song traits typical of D. pseudoobscura (short interpulse interval or low intrapulse frequency), and those that failed to mate to have song traits similar to D. persimilis. We verified our conclusions with several other types of analyses, but the conclusions were always identical to those derived from the Mann-Whitney $U$-test.

\section{Results}

\section{Pure species songs}

As documented previously (Waldron, 1964; Ewing, 1969; Noor \& Aquadro, 1998), the courtship songs of $D$. pseudoobscura and $D$. persimilis differ substantially from each other. No overlap between species in either median interpulse interval (IPI) or median intrapulse 
frequency was noted. The median value among D. pseudoobscura males for median IPI was $40.45 \mathrm{~ms}$, with a range of $38.35 \mathrm{~ms}$ to $45.03 \mathrm{~ms}$ among males. D. persimilis males expressed a median IPI of $54.66 \mathrm{~ms}$ with a range of $47.74 \mathrm{~ms}$ to $60.10 \mathrm{~ms}$ among males. The median value among $D$. pseudoobscura males for intrapulse frequency was $259 \mathrm{~Hz}$ with a range of $223 \mathrm{~Hz}$ to $357 \mathrm{~Hz}$. In contrast, D. persimilis songs possessed a median frequency of $446 \mathrm{~Hz}$ with a range of $402 \mathrm{~Hz}$ to $515 \mathrm{~Hz}$.

\section{Genetics of species song differences}

Both of the two song traits analysed appear to be influenced by numerous genetic differences between D. pseudoobscura and D. persimilis (see Table 1, Figs 3 and 4). Differences between these species in interpulse interval (IPI) mapped to loci on the left arm of the $\mathrm{X}$-chromosome (DPSX004), the right arm of the $\mathrm{X}$-chromosome (DPSX009), the centre of the second chromosome, and the third chromosome, when evaluated using single marker regressions. Except for the third chromosome, all of these regions were strongly associated with IPI when evaluated using composite interval mapping analysis as well, and the effect mapped to the region of DPS2002 along the second chromosome (Fig. 3).
Differences between D. pseudoobscura and D. persimilis in intrapulse frequency were also initially localized to the left arm of the X-chromosome, the second chromosome, and the third chromosome by single marker regressions (Table 1). Only the regions of DPSX004 and DPS2002 were significantly associated with species differences in intrapulse frequency when evaluated with composite interval mapping analyses (Fig. 4). No significant species courtship song differences were associated with loci on the fourth or fifth chromosomes.

Environmental variance was estimated in pure species songs because no genetic variance would exist in the inbred lines used. We estimated environmental variance in interpulse interval to be $9.4 \mathrm{~ms}^{2}$, and the environmental variance in intrapulse frequency to be $1370 \mathrm{~Hz}^{2}$ in pure species songs. The phenotypic variances of backcross progeny songs were $15 \mathrm{~ms}^{2}$ for IPI and $5397 \mathrm{~Hz}^{2}$ for frequency. Using these figures, we determined maximum estimates of broad sense heritability to be 0.37 for IPI and 0.75 for frequency in backcross males. The difference between the heritabilities of these song elements is consistent with previous observations in these species (Noor \& Aquadro, 1998).

Using our heritability estimates, we calculated a minimum proportion of genetic variance associated with particular QTLs identified by composite interval mapping (Table 2). We find that almost all of the

\begin{tabular}{|c|c|c|c|c|}
\hline \multirow[b]{2}{*}{ Marker } & \multicolumn{2}{|c|}{ Intrapulse frequency } & \multicolumn{2}{|c|}{ Interpulse interval } \\
\hline & $r^{2}$ & $P$ & $r^{2}$ & $P$ \\
\hline \multicolumn{5}{|l|}{ X-chromosome } \\
\hline$D P S X 004$ & 0.103 & $<0.0001 *$ & 0.190 & $<0.0001 *$ \\
\hline$D P S X 009$ & 0.005 & 0.1240 & 0.140 & $<0.0001^{*}$ \\
\hline \multicolumn{5}{|l|}{ 2nd chromosome } \\
\hline DPS2005 & 0.018 & 0.0100 & 0.024 & $<0.0001^{*}$ \\
\hline DPS2001 & 0.200 & $<0.0001 *$ & 0.115 & $<0.0001^{*}$ \\
\hline DPS2002 & 0.240 & $<0.0001 *$ & 0.140 & $<0.0001^{*}$ \\
\hline DPS2006 & 0.140 & $<0.0001 *$ & 0.077 & $<0.0001 *$ \\
\hline Gld & 0.029 & $<0.0001 *$ & 0.013 & 0.0120 \\
\hline DPS2003 & 0.008 & 0.0678 & 0.001 & 0.4154 \\
\hline \multicolumn{5}{|l|}{ 3rd chromosome } \\
\hline DPS3004 & 0.018 & $0.0012 *$ & 0.027 & $<0.0001^{*}$ \\
\hline DPS3002 & 0.026 & $<0.0001 *$ & 0.012 & 0.0292 \\
\hline DPS3005 & 0.035 & $0.0002 *$ & 0.000 & 0.8822 \\
\hline \multicolumn{5}{|l|}{ 4th chromosome } \\
\hline DPS4003 & 0.000 & 0.8356 & 0.000 & 0.5926 \\
\hline$A d h$ & 0.008 & 0.0550 & 0.016 & 0.0082 \\
\hline DPS4002 & 0.000 & 0.8050 & 0.001 & 0.3276 \\
\hline $\begin{array}{l}\text { 5th chromosome } \\
\text { eyeless }\end{array}$ & 0.003 & 0.2688 & 0.012 & 0.0184 \\
\hline
\end{tabular}

Table 1 Association of genetic markers with differences intrapulse frequency and interpulse interval in backcross hybrids of Drosophila pseudoobscura and D. persimilis

${ }^{*} P<0.05$ after Bonferroni correction. 
Fig. 3 Logarithm of odds (LOD) scores for associations of various positions along the second and third chromosomes with interpulse interval. Dashed lines indicate the LOD score required for statistical significance in the composite interval mapping analysis. Solid lines indicate the composite interval mapping LOD scores. Diamonds indicate the single marker regression LOD scores.
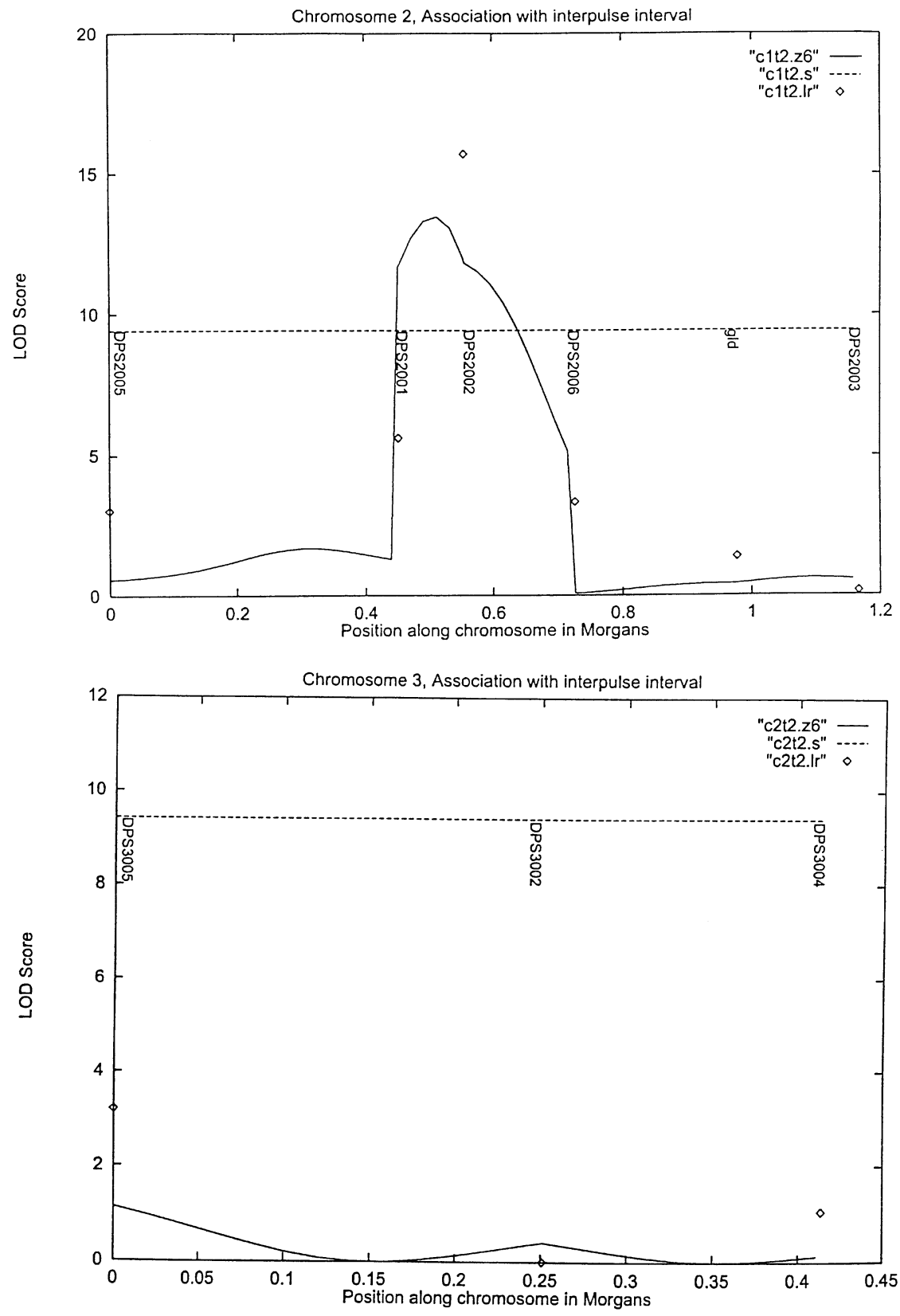

estimated genetic variance in IPI is associated with the three significant QTLs identified in this study. About one-third of the genetic variance in frequency is associated with the two significant QTLs we identified for this character.

\section{Relationship of song parameters to mating success}

We tested whether backcross hybrid male mating success to $D$. pseudoobscura or D. persimilis females was associated with courtship song IPI or frequency. Backcross males that mated successfully with $D$. pseudo- obscura females sang with a significantly lower median IPI than those males that failed to mate $(P<0.0001$, Table 3). No significant difference in intrapulse frequency was noted between males that mated with D. pseudoobscura females and males that did not. In contrast, males that mated successfully with $D$. persimilis females sang with a significantly higher median frequency than those males that failed to mate $(P=0.0058$, Table 3$)$. No difference in IPI was noted between males that mated with $D$. persimilis females and males that did not. Hence, frequency is significantly associated with backcross male mating success to

(c) The Genetics Society of Great Britain, Heredity, 86, 68-77. 

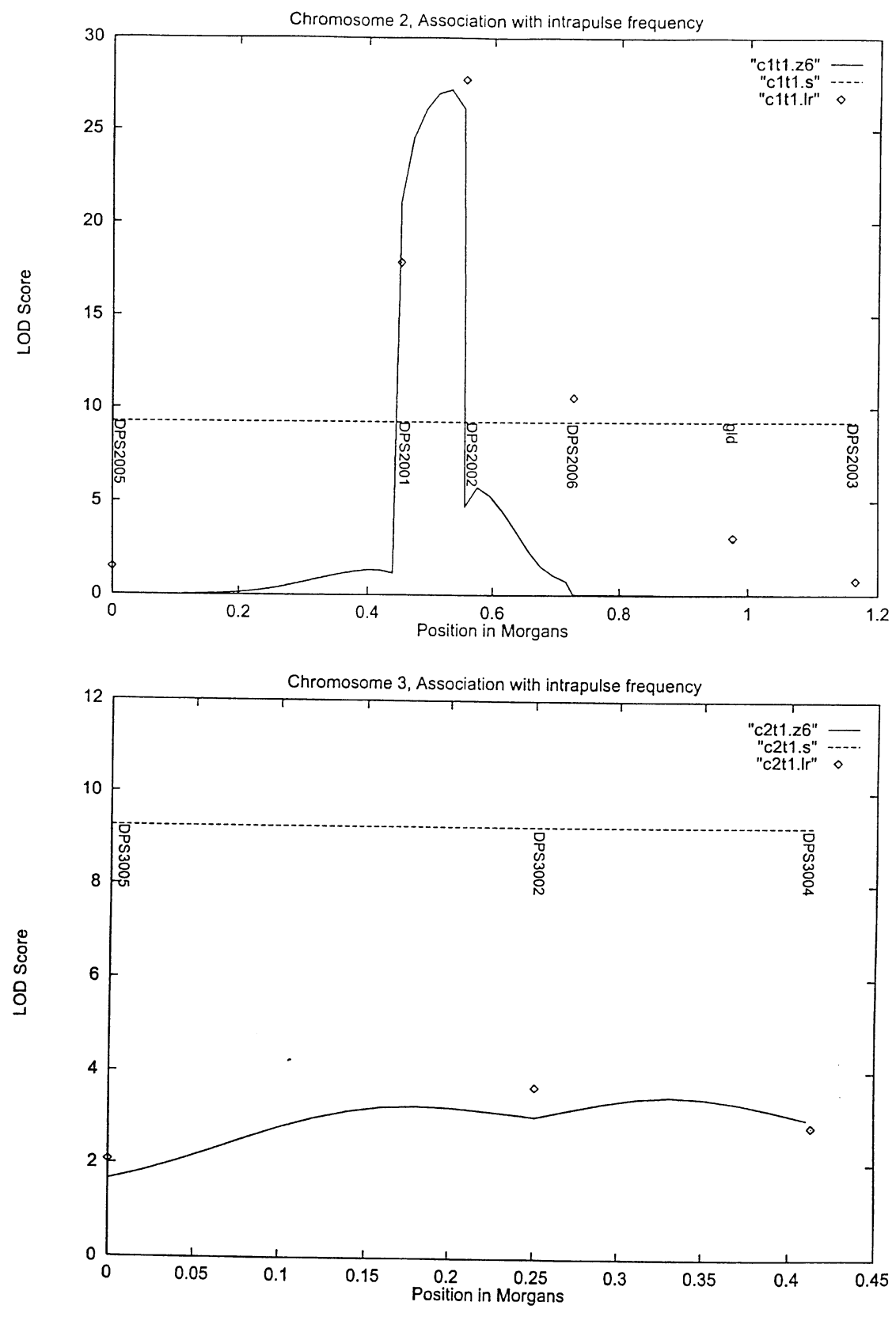

Fig. 4 LOD scores for associations of various positions along the second and third chromosomes with intrapulse frequency.

$D$. persimilis females, whereas IPI is significantly associated with backcross male mating success to $D$. pseudoobscura females.

\section{Discussion}

We have mapped the genetic basis of two differences in courtship song between Drosophila pseudoobscura and $D$. persimilis to three of the four major chromosome arms. Our observations are in accord with results from other investigators: differences among Drosophila males in courtship song parameters are typically controlled by multiple genes (e.g. Pugh \& Ritchie, 1996; Hoikkala et al., 2000). Similarly, we have identified an association between courtship song interpulse interval (IPI) and mating success to D. pseudoobscura females, as well as an association between song intrapulse frequency and mating success to $D$. persimilis females. These associations are consistent with an earlier suggestion that females of these species do not rely on the same phenotypic cues to preferentially select conspecific mates (Noor, 1997b).

A study of courtship song by Noor \& Aquadro (1998) failed to find an association between IPI and mating

(c) The Genetics Society of Great Britain, Heredity, 86, 68-77. 
Table 2 Proportions of phenotypic and genetic variance in Drosophila associated with particular QTLs that were significantly associated with song parameters in a composite interval mapping analysis

\begin{tabular}{|c|c|c|c|c|}
\hline \multirow[b]{2}{*}{ QTL } & \multicolumn{2}{|c|}{$\begin{array}{l}\text { Intrapulse } \\
\text { frequency }\end{array}$} & \multicolumn{2}{|c|}{$\begin{array}{l}\text { Interpulse } \\
\text { interval }\end{array}$} \\
\hline & $\% V_{\mathrm{P}}$ & $\% V_{\mathrm{G}}$ & $\% V_{\mathrm{P}}$ & $\% V_{\mathrm{G}}$ \\
\hline $\begin{array}{l}\text { X-chromosome } \\
\text { QTL at DPSX004 } \\
\text { QTL at DPSX009 }\end{array}$ & $\begin{array}{l}5.1 \% \\
\text { NS }\end{array}$ & $\begin{array}{l}6.8 \% \\
\text { NS }\end{array}$ & $\begin{array}{l}13.9 \% \\
12.7 \%\end{array}$ & $\begin{array}{l}37.3 \% \\
34.1 \%\end{array}$ \\
\hline $\begin{array}{l}\text { 2nd chromosome } \\
\text { QTL near } \\
\text { DPS2002 }\end{array}$ & $22.4 \%$ & $30.0 \%$ & $9.1 \%$ & $24.4 \%$ \\
\hline
\end{tabular}

NS, not significant.

success to D. pseudoobscura females. However, the sample size used by the study reported here, and correspondingly its power for detecting associations, was much higher. More direct evidence for a role of courtship song parameters in species mating discrimination in both of these taxa is forthcoming from studies using playback of recorded song and courtship by wingless males (Mark Lineham and Michael Ritchie, unpublished data).

From the mapping results presented here, we can estimate the minimum number of genes that contribute to the species differences in IPI and intrapulse frequency. Clearly, at least three genes contribute to the species difference in IPI and at least two genes contribute to the species difference in frequency. Although these minimum estimates are informative, the species differences in song characters may result from differences at more genes. The strongest associations of each of these phenotypes with markers always involved markers within segments of the genome that are inverted between the two species (DPSX004, DPS2002, and in one case $D P S X 009$ ). This association may result from an ascertainment bias because recombination is effectively inhibited between QTLs and markers within inversions.
However, we suggest that song character differences between these species may not be highly polygenic, or at least not highly polygenic and randomly distributed throughout the genome. In the case of courtship song IPI, the three QTLs we identified within inverted regions were associated with effects totalling to nearly all the estimated genetic variance in IPI. This finding suggests that the remainder of the genome contributes little or no effect to IPI differences between these species.

Similarly, we failed to detect a significant association between the inverted right arm of the X-chromosome (XR) and the difference between these species in intrapulse frequency. The XR inversion spans approximately 11 cytological bands, and the inhibition of recombination appears to extend across almost the entire chromosome arm (Noor \& Smith, 2000; J. Hey, personal communication). This chromosome arm constitutes approximately $20 \%$ of the genome of these species. With the sample size used in this study, we would have greater than a $95 \%$ chance of detecting a QTL contributing $20 \%$ of the genetic variance in this trait, assuming additivity of courtship song differences between these species. The lack of any detectable effect from this chromosome arm given our large sample size suggests that this large portion of the genome contributes little or nothing to courtship song frequency differences between the species. It is therefore improbable that frequency is both polygenic and controlled by factors spread randomly across the genome. Song differences between these species appear to be primarily controlled by a moderate number of genes contributing large effects (or several genes clustered within regions inverted between these species).

There were differences between our results obtained from single marker linear regressions and composite interval mapping with regard to effects of the third chromosome. The deviation of DPSX004 alleles from the expected 1:1 ratio among backcross males may have biased the single marker association tests for these third chromosome loci. Although third chromosome marker alleles were observed among backcross males at the
Table 3 Association in Drosophila of backcross hybrid male mating success with courtship song parameters

\begin{tabular}{|c|c|c|c|}
\hline & Number & Intrapulse frequency & Interpulse interval \\
\hline \multicolumn{4}{|c|}{ Males paired with $D$. persimilis } \\
\hline Mated & 109 & $309 \mathrm{~Hz}(185-741)$ & $40.5 \mathrm{~ms}(34.2-48.4)$ \\
\hline Failed to mate & 137 & $281 \mathrm{~Hz}(196-565)^{*}$ & $40.2 \mathrm{~ms}(33.0-53.0)$ \\
\hline \multicolumn{4}{|c|}{ Males paired with $D$. pseudoobscura } \\
\hline Mated & 195 & $284 \mathrm{~Hz}(159-595)$ & $40.2 \mathrm{~ms}(33.4-54.0)$ \\
\hline Failed to mate & 51 & $303 \mathrm{~Hz}(198-432)$ & $44.5 \mathrm{~ms}(34.9-55.9)^{* *}$ \\
\hline
\end{tabular}


expected 1:1 ratio, their association with alleles of DPSX004 was nonrandom as determined by a chisquared test (e.g. $P=0.003$ for DPS3004). This association would bias single marker association tests, but composite interval mapping corrected for it.

Several loci that affect male courtship song have been identified in D. melanogaster (e.g. Peixoto \& Hall, 1998). We intend to evaluate the role of these loci in contributing to the differences between $D$. pseudoobscura and D. persimilis in their courtship song parameters. We also intend to analyse the nature of courtship song variation within these species more thoroughly (e.g. Noor et al., 2000b). Finally, we intend to introgress genomic regions between these species (outside the inversion differences) and evaluate whether and how many other loci may contribute to the species differences in courtship song.

\section{Acknowledgements}

We thank L. Bertucci, K. Grams, A. Hartzog, K. Nettles, B. Rogge, K. Smith, and N. Tuminello for technical assistance. J. Brookfield, N. Espinoza, H. McGuire, J. Reiland, B. Rogers, and two anonymous reviewers provided helpful comments on the manuscript. Courtship song analyses were performed using CANARY software (version 1.2.4) from the Cornell Laboratory of Ornithology, Bioacoustics Research Program. This research has been supported by a Sigma Xi grant-in-aid of research to M.A.W. and National Institutes of Health (NIH) grant GM58060 subcontracted through J. Hey at Rutgers University to M.A.F.N. M.A.W. was supported by Howard Hughes Medical Institute undergraduate research fellowships from 1998 to 2000 .

\section{References}

BASTEN, C. J., WEIR, B. S. AND ZENG, Z.-B. 1999. QTL CARTOGRAPHER: a reference manual and tutorial for QTL mapping, version 1.13. Department of Statistics, North Carolina State University, Raleigh, NC.

CHANG, H.-C. AND MILlER, D. D. 1978. Courtship and mating sounds in species of the Drosophila affinis subgroup. Evolution, 32, 540-550.

COWling, D. E. AND BURNeT, B. 1981. Courtship songs and genetic control of their acoustic characteristics in sibling species of the Drosophila melanogaster subgroup. Anim. Behav., 29, 924-935.

COYNE, J. A., CRITTENDEN, A. P. AND MAH, K. 1994. Genetics of a pheromonal difference contributing to reproductive isolation in Drosophila. Science, 265, 1461-1464.

DOBZHANSKy, T. 1936. Studies of hybrid sterility. II. Localization of sterility factors in Drosophila pseudoobscura hybrids. Genetics, 21, 113-135.
EWING, A. W. 1969. The genetic basis of sound production in Drosophila pseudoobscura and D. persimilis. Anim. Behav., 17, 555-560.

EWING, A. W. AND BENNET-CLARK, H. C. 1968. The courtship songs of Drosophila. Behaviour, 31, 288-301.

GENG, Z., ZHU, D., SUN, Y., CAI, H., CHEN, J. AND ZHU, C. 1989. Study on the courtship song of six species in Drosophila takahashii subgroup. Acta Genet. Sin., 16, 448-454.

GLEASON, J. M. AND RITCHIE, M. G. 1998. Evolution of courtship song and reproductive isolation in the Drosophila willistoni species complex: Do sexual signals diverge the most quickly? Evolution, 52, 1493-1500.

GLOOR, G. B., PRESTON, C. R., JOHNSON-SCHLITZ, D. M., NASSIF, N. A., Phillis, R. W. ET AL. 1993. Type I repressors of P element mobility. Genetics, 135, 81-95.

GORCZYCA, M. AND HALL, J. C. 1987. The INSECTAVOX, an integrated device for recording and amplifying courtship song. Drosoph. Inf. Serv., 66, 157-160.

HAMILTON, M. B., PINCUS, E. L., DIFIORE, A. AND FLEISCHER, R. C. 1999. Universal linker and ligation procedures for construction of genomic DNA libraries enriched for microsatellites. Biotechniques, 27, 500-507.

HOIKKALA, A., ASPI, J. AND SUVANTO, L. 1998. Male courtship song frequency as an indicator of male genetic quality in an insect species, Drosophila montana. Proc. Roy. Soc. B, 265, 503-508.

HOIKKALA, A., KANESHIRO, K. Y. AND HOY, R. R. 1994. Courtship songs of the picture-winged Drosophila planitibia subgroup species. Anim. Behav., 47, 1363-1374.

hoikKala, A., PaAllysaho, S., ASPI, J. AND lumme, J. 2000. Localization of genes affecting species differences in male courtship song between Drosophila virilis and D. littoralis. Genet. Res., 75, 37-45.

KAWANISHI, M. AND WATANABE, T. K. 1981. Genes affecting courtship song and mating preference in Drosophila melanogaster, Drosophila simulans, and their hybrids. Evolution, 35, 1128-1133.

KYRIACOU, C. P. AND HALL, J. C. 1982. The function of courtship song rhythms in Drosophila. Anim. Behav., 30, 794-801.

MERRELl, D. J. 1954. Sexual isolation between Drosophila persimilis and Drosophila pseudoobscura. Am. Nat., 88, 93-99.

NOOR, M. A. 1995. Speciation driven by natural selection in Drosophila. Nature, 375, 674-675.

NOOR, M. A. F. 1996. Absence of species discrimination in Drosophila pseudoobscura and D. persimilis males. Anim. Behav., 52, 1205-1210.

NOOR, M. A. F. 1997a. Environmental effects on male courtship intensity in Drosophila pseudoobscura (Diptera: Drosophilidae). J. Insect Behav., 10, 305-312.

NOOR, M. A. F. 1997b. Genetics of sexual isolation and courtship dysfunction in male hybrids of Drosophila pseudoobscura and D. persimilis. Evolution, 51, 809-815.

NOOR, M. A. F. AND AQUADRo, C. F. 1998. Courtship songs of Drosophila pseudoobscura and D. persimilis: analysis of variation. Anim. Behav., 56, 115-125.

NOOR, M. A. F. AND COYNE, J. A. 1996. Genetics of a difference in cuticular hydrocarbons between Drosophila pseudoobscura and D. persimilis. Genet. Res., 68, 117-123.

(c) The Genetics Society of Great Britain, Heredity, 86, 68-77. 
NOOR, M. A. F. AND SMITH, K. R. 2000. Recombination, statistical power, and genetic studies of sexual isolation in Drosophila. J. Hered., 91, 99-103.

NOOR, M. A. F., SCHUG, M. D. AND AQUADRO, C. F. 2000a. Microsatellite variation in populations of Drosophila pseudoobscura and Drosophila persimilis. Genet. Res., 75, 25-35.

NOOR, M. A. F., WILliAMS, M. A., ALVAREZ, D. AND RUIZ-GARCíA, M. 2000b. Lack of evolutionary divergence in courtship songs of Drosophila pseudoobscura subspecies. J. Insect Behav., 13, 255-262.

ORR, H. A. 1987. Genetics of male and female sterility in hybrids of Drosophila pseudoobscura and D. persimilis. Genetics, 116, 555-563.

PAlumbI, S. R. 1996. Nucleic acids II: The polymerase chain reaction. In: Hillis, D. M., Moritz, C. and Mable, B. K. (eds) Molecular Systematics, pp. 205-247. Sinauer Associates, Sunderland, MA.

PEIXOTO, A. A. AND HALl, J. C. 1998. Analysis of temperaturesensitive mutants reveals new genes involved in the courtship song of Drosophila. Genetics, 148, 827-838.

PUGH, A. R. G. AND RITCHIE, M. G. 1996. Polygenic control of a mating signal in Drosophila. Heredity, 77, 378-382.

RICE, W. R. 1989. Analyzing tables of statistical tests. Evolution, 43, 223-225.

RITCHIE, M. G. AND GLEASON, J. M. 1995. Rapid evolution of courtship song pattern in Drosophila willistoni sibling species. J. Evol. Biol., 8, 463-479.
RITCHIE, M. G., TOWNHILL, R. M. AND HOIKKALA, A. 1998. Female preference for fly song: Playback experiments confirm the targets of sexual selection. Anim. Behav., 56, 713-717.

RITCHIE, M. G., HALSEY, E. J. AND GLEASON, J. M. 1999. Drosophila song as a species-specific mating signal and the behavioural importance of Kyriacou \& Hall cycles in D. melanogaster song. Anim. Behav., 58, 649-657.

STENNETT, M. D. AND ETGES, W. J. 1997. Premating isolation is determined by larval rearing substrates in cactophilic Drosophila mojavensis. III. Epicuticular hydrocarbon variation is determined by use of different host plants in Drosophila mojavensis and Drosophila arizonae. J. Chem. Ecol., 23, 2803-2824.

TOMARU, M. AND OGUMA, Y. 1994. Genetic basis and evolution of species-specific courtship song in the Drosophila auraria complex. Genet. Res., 63, 11-17.

TOMARU, M., MATSUBAYASHI, H. AND OGUMA, Y. 1995. Heterospecific inter-pulse intervals of courtship songs elicit female rejection in Drosophila biauraria. Anim. Behav., 50, 905-914.

WALDRON, I. 1964. Courtship sound production in two sympatric sibling Drosophila species. Science, 144, 191-193.

WANG, R. L., WAKELEY, J. AND HEY, J. 1997. Gene flow and natural selection in the origin of Drosophila pseudoobscura and close relatives. Genetics, 147, 1091-1106. 